\title{
Circuit
}

Musiques contemporaines

\section{Le NEM a 30 ans, et « il faut continuer. De cela, je n'ai aucun doute. "}

\section{Paul Bazin}

Volume 30, numéro 1, 2020

URI : https://id.erudit.org/iderudit/1069086ar

DOI : https://doi.org/10.7202/1069086ar

Aller au sommaire du numéro

Éditeur(s)

Circuit, musiques contemporaines

ISSN

1183-1693 (imprimé)

1488-9692 (numérique)

Découvrir la revue

Citer ce document

Bazin, P. (2020). Le NEM a 30 ans, et « il faut continuer. De cela, je n'ai aucun doute. ». Circuit, 30(1), 81-84. https://doi.org/10.7202/1069086ar d'utilisation que vous pouvez consulter en ligne.

https://apropos.erudit.org/fr/usagers/politique-dutilisation/ 


\title{
Le NEM a 30 ans, et «il faut continuer. De cela, je n’ai aucun doutel. »
}

\author{
Paul Bazin ${ }^{2}$
}

Au moment de célébrer un anniversaire, quoi de plus normal que de marquer un temps d'arrêt pour se retourner et contempler, derrière soi, le travail accompli? Pour prendre état du chemin parcouru avant de regarder à nouveau vers l'avant, de plonger, tête baissée, vers des lendemains parfois espérés, souvent inattendus. Faire un pas de côté, en somme, dans un geste qui a tout de la mise en abîme: la fondation du Nouvel Ensemble Moderne n'était-elle

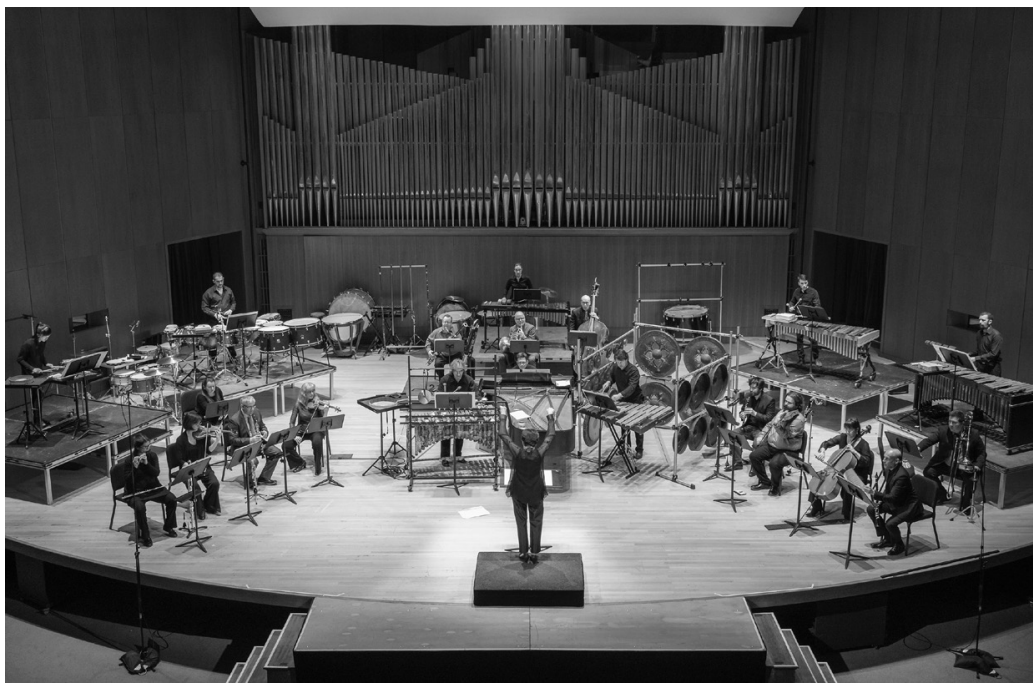

Grand concert $30^{\mathrm{e}}$ anniversaire du NEM, 3 mai 2019, salle Claude-Champagne, Montréal. Sous la direction de Lorraine Vaillancourt, avec la participation de l'ensemble à percussion Sixtrum. pas elle-même, de fait, un magnifique pas de côté? Un contrepoids proposé par la volonté d'excellence au rythme effréné des productions de l'art? L'apologie du temps consacré, de la lenteur.

Laissant toute la place à la musique et aux musiciens, Lorraine Vaillancourt impressionne néanmoins par la qualité exceptionnelle d'une direction musicale qui conjugue précision, intelligence et sensibilité. Sans doute mieux que personne, elle assume la lourde succession d'un Serge Garant.

- Carol Bergeron Le Devoir, 5 mai 1989.

Par un heureux paradoxe, le NEM s'est imposé comme un ensemble de pointe, de stature internationale, à une vitesse vertigineuse. En novembre 1989, quelques mois à peine suivant le concert inaugural (salle ClaudeChampagne, le 3 mai 1989, soit il y a très précisément 30 ans ce soir), ses musicien.nes se rendaient à
New York pour y faire entendre, entre autres, Ligeti, Benjamin et Berio... «an impressive New York debut», pour reprendre les mots du critique Allan Kozinn ${ }^{3}$. Laccueil du concert fut à ce point enthousiaste que huit mois plus tard, le NEM se rendait à nouveau dans la métropole étatsunienne pour y faire entendre, cette fois, un programme constitué essentiellement d’œuvres de compositeurs québécois: Mather, Rose et Longtin; Evangelista, Rea et Vivier, aussi. La présence de ces

Le Nouvel Ensemble Moderne seems intent on making its name quickly. The group [...] was founded in Montreal six months ago. It gave its first New York concert on Saturday afternoon at Weill Recital Hall, with performances as polished as one would expect from collaboration of much longer standing.

- Allan Kozinn The New York Times, 15 novembre 1989. 
On avait envie de proposer autre chose - pas seulement de la musique, mais toutes sortes d'événements mélangeant parfois danse, arts visuels, lecture de textes, thêâtre... C'est comme ça que les Événements du neuf (les 9 du mois à neuf heures, dans des salles différentes chaque fois) sont nés.

- Lorraine Vaillancourt Circuit, vol. 23, $n^{0} 3,2013$

derniers sur la liste n'est d'ailleurs pas fortuite, puisqu'ils furent tous trois les compagnons d'armes de Lorraine Vaillancourt aux premières heures d'une aventure à la fois entière et déjà porteuse de l'impulsion qui mena à la fondation du NEM : celle des Événements du neuf (1978-1990), qui durant douze années furent euxmêmes un pôle important de la vie musicale contemporaine de Montréal.

En retrait de l'épicentre qui, au tournant des années 1980, voit naître plusieurs des grands ensembles européens voués à la musique contemporaine, l'apparition du NEM a ceci de particulier qu'elle s'inscrit à leur suite tout en les devançant. Le NEM a en effet de quoi prétendre au titre de prédécesseur, si l'on compte sur son enracinement dans le terreau exploratoire des Événements du neuf, par seulement deux ans cadets de l'incontournable Ensemble intercontemporain, fondé à Si le premier mandat du NEM était de jouer les "classiques" du XX $x^{e}$ siècle, là on était propulsé dans la jeune création, et ces jeunes compositeurs, toujours très heureux de leur expérience montréalaise qu'ils traversent comme un rêve, ont été nos meilleurs ambassadeurs.

- Lorraine Vaillancourt Circuit, vol. 23, $n^{\circ} 3,2013$. permet d'assister à toute une série de concerts consacrés à un artiste de renom ou aux créateurs d'une région du monde - la première Biennale fut d'abord consacrée à Kagel, en 1992, puis ce fut le tour de Donatoni (1994) et des représentants des pays scandinaves (1997) - tandis qu'une autre année, c'est la jeune génération qui est mise à l'honneur. Au cours des 13 éditions du Forum (la dernière s'est tenue en 2016), ce sont des dizaines de compositrices et de compositeurs qui ont été invitées à venir travailler avec le NEM dans le contexte d'une formule intensive, parfois thématique (musique mixte [2008] et musique et vidéo d'art [2010]): trois à quatre semaines de travail, de conférences, de tables rondes et de concerts. Une occasion souvent unique d'acquérir, en un court laps de temps, une expérience professionnelle d'une si grande rigueur. Parmi les événements qui contribuèrent à Paris en 1976 par Pierre Boulez.

De cette première initiative, le NEM retient également tant le désir de défendre les «classiques» du $\mathrm{xx}^{\mathrm{e}}$ siècle que d'offrir une voix rigoureuse et expérimentée aux créateurs de la relève. Les Biennales et les différentes éditions du Forum international des jeunes compositeurs alternent ainsi au cours de la première décennie de l'ensemble. Une année, la Biennale

À travers ce parcours musical d'une soirée, à la salle ClaudeChampagne, le NEM a donné à la fois le meilleur de lui-même et le meilleur de la Scandinavie. Mettant en relief chaque détail de ces œuvres, avec finesse mais non sans énergie, tout en maintenant une sonorité d'ensemble éblouissante de clarté. - Dominique Olivier Voir, 20 au 26 novembre 1997.

l'essor fulgurant du NEM au cours de ses premières années, la captation que Radio-Canada réalisa du tout premier Forum (1991) revêt d'ailleurs une importance toute particulière, tant son rayonnement à l'étranger fut important. De là, il n’y avait évidemment plus qu'un pas à faire, en toute cohérence, pour que soient mises sur pied les Rencontres de musique nouvelle du Domaine Forget, en 1994. D’abord destinées aux compositrices et compositeurs et marquées par la présence de mentors de la trempe d'un Jonathan Harvey ou d'un Pascal Dusapin, ces rencontres s'ouvrent rapidement aux instrumentistes, élargissant dès lors le spectre des visées de l'ensemble du seul domaine de la création à tout le champ de la relève en musique nouvelle. Le fait 
que le NEM soit en résidence à la Faculté de musique de l'Université de Montréal depuis ses débuts n'est pas non plus sans rappeler ces objectifs, puisque grâce au travail rapproché avec les compositeurs, et peut-être plus encore à travers la proximité avec les interprètes de demain, on cherche à inscrire le répertoire dans la durée, de même que sa pratique et la maîtrise instru-
Je n'ai retenu qu'une seule leçon, la première, des quelque dix séminaires que Pascal Dusapin a donnés à l'été 2002, dans le cadre du stage de musique nouvelle du Domaine Forget organisé par Denys Bouliane et le Nouvel Ensemble Moderne. [...] alors que la leçon se terminait, moi, je naissais à la création pour toujours. - Julien Bilodeau Circuit, vol. 29, $n^{\circ}$ 1, 2019. mentale qu'elle exige. C'est inscrit dans l'ADN même de l'ensemble: comment négliger l'importance des interprètes dans la transmission des œuvres au public?

Dans son rapport singulier au temps, le NEM a d'ailleurs su s'attacher bon nombre de ses musiciens et musiciennes pour une durée épatante! On s'étonne avec joie de l'incroyable longévité des instrumentistes au sein de l'ensemble. De la quinzaine d'individus qu'il comprenait au départ moins l'irréductible Lorraine -, le NEM peut toujours compter, 30 ans plus tard, sur trois de ses membres fondateurs. Toutes proportions gardées, n'est-ce pas admirable? Et c'est encore sans mentionner celles et ceux qui s'y sont joints.es au fil du temps et qui, dans de nombreux cas, cumulent plusieurs années, voire une ou deux décennies de compagnonnage et de dévouement passionné! Et que dire de Normand Forget, fidèle hautboïste au sein de l'ensemble pendant plus de vingt-cinq ans, qui en a repris la direction artistique et générale ces dernières années! En acteurs et actrices et témoins d'un historique qui tend vers demain, les musiciens et musiciennes du NEM sont peut-être ceux

Après tout ce temps, le plaisir de se rencontrer est encore bien présent... l'atmosphère est propice comme elle l'a toujours été... Dès le tout début du NEM, la musique avait découvert de joyeux complices. Un creuset pour une musique qui exige beaucoup!

- Julien Grégoire Un parcours contemporain, 2014. et celles qui, avec le plus d'éloquence, nous rappellent en mémoire les milliers d'heures consacrées à la mise au monde d'œuvres peu jouées ou carrément inconnues; les milliers de kilomètres avalés pour porter de par le monde ces musiques qui ne demandent qu'à s'incarner; les projets réalisés en coulisse, si nombreux qu'il est impossible de tous les nommer ici.

On se souviendra néanmoins des nombreux voyages effectués par le NEM: les tournées, entreprises dès 1992 avec l'Europe, lesquelles ont mené l'ensemble en tant de lieux: Allemagne, Angleterre, Australie, Belgique, Canada, Chine,
Un chef sans musiciens est plus misérable qu'un musicien sans chef.

- Lorraine Vaillancourt Discours de réception à la Société royale du Canada, 1998. Espagne, États-Unis, France, Italie, Japon, Mexique, Pays-Bas, Singapour... Trois Forums, aussi, que le NEM, sur invitation de partenaires étrangers, a tenus en Australie (2000), aux Pays-Bas (2006) et en France (2008). On pensera encore aux disques, pour certains réalisés au Canada, pour d'autres à l'étranger, comme en témoignent les enregistrements d'œuvres québécoises (Musiques de Montréal, 1991; Bouliane Gougeon - Rea, 2012) et les projets monographiques (Harvey, 1996 et 2000; Carter, 2001; de Pablo, 2005). Le dernier disque en date du NEM (Dubedout - Leroux: Tournoiements, 2018) rappelle d'ailleurs comment certains projets d'enregistrements sont étroitement associés aux séjours du NEM à l'étranger, celui-ci ayant été réalisé à l'occasion du passage de l'ensemble à Toulouse, à l'automne 2017. Le Diapason d'or attribué au disque sur lequel fut enregistré Bhakti, de Harvey, de même que la collection complète des prix Opus décernés par le Conseil québécois de la musique pour tel ou tel disque, tel ou tel événement, rendent aussi bien compte de l'accueil chaleureux que rencontrent les projets du NEM au fil des ans. De l'arrangement de Wozzeck par John Rea, en 1995 (repris à l'occasion du concert $25^{\mathrm{e}}$ anniversaire, à la Maison symphonique), à la commande d'une œuvre à Tristan Murail (2009), 
Les musiciens dU NEM nous ont offert une performance de grande qualité où rien n'était laissé au hasard. Mentionnons particulièrement la variété des timbres, la précision rythmique, la souplesse de l'expression et un registre de nuances proche de celui de l'orchestre.

$$
\text { - Mayumi Tsuji }
$$

Ongaku Buyo Shimbu, 1996.

de Vivier à Leroux, en passant par la réalisation d'un événement soulignant les 50 ans du Refus global (1999) et par les multiples collaborations (avec Chants Libres, pour n'en nommer qu'une), on ne peut qu'applaudir devant l'incroyable fécondité de tous ces projets que l'ensemble est parvenu à réaliser alors même qu'il s'imposait - et s'impose toujours - d'y accorder plus de temps que nul autre n'y consentirait.

« [I]l faut continuer. De cela, je n’ai aucun doute.» Ces mots de Lorraine Vaillancourt - qui terminaient une entrevue accordée à Jonathan Goldman en 2013 - conservent aujourd'hui toute leur pertinence, maintiennent leur importance. À l'heure du quantifiable à tout prix. À l'heure du rendement. À l'heure où le risque, l'exigeant et l'inconnu ont souvent mauvaise presse, le NEM, par son engagement vital à défendre les classiques contemporains tout autant que les plus récentes créations - qu'il contribue à inscrire à son tour au rang des nouveaux classiques!-, nous garde de tous ces fous éteignoirs de la culture. Pour tout ce qui a été fait, et pour tout ce que l'avenir nous garde en réserve d'accomplissements remarquables: chapeaux bas!

\section{B I B L I O G RA P H I E}

Bergeron, Carol (1989), «Naissance d'une nouvelle formation éblouissante», Le Devoir, 5 mai.

Goldman, Jonathan (2013), «Donner le temps: entretien avec Lorraine Vaillancourt», Circuit, musiques contemporaines, vol. $23, n^{\circ} 3$, p. 49-62.

Kozinn, Allan (1989), «Nouvel Ensemble Moderne Makes New York Debut», The New York Times, 15 novembre.

Kozinn, Allan (1990), «Works From Quebec by Le Nouvel Ensemble [sic]», The New York Times, $1^{\text {er }}$ juillet.

Majeau-Bettez, Emanuelle (2019), «Rencontres et “émotions nouvelles”: Pascal Dusapin raconté par cinq collaborateurs », avec la participation de Barbara Hannigan, Samy Moussa, Armand Angster, Julie-Anne Derome et Julien Bilodeau, Circuit, musiques contemporaines, vol. 29, n ${ }^{\circ}$ 1, p. 51-62.

Olivier, Dominique (1997), «Étoiles du Nord. Scandinavie: Biennale 1997 du Nouvel Ensemble Moderne», Voir, 20 au 26 novembre.

Tremblay, Raynald (1996), «Wozzeck par le Nouvel Ensemble Moderne », Spirale, janvier-février, p. 21.

Tsuji, Mayumi (1996), «Le Nouvel Ensemble Moderne Made a High Level Performance» [traduit du japonais par le Service de traduction de l'ambassade canadienne], Ongaku Buyo Shimbun, $1^{\mathrm{er}}$ mai.

VAILlanCOURT, Lorraine (1998), «La musique aujourd'hui dans tous ses états ", Circuit, musiques contemporaines, vol. 9, $\mathrm{n}^{\circ} 2$, p. 75 -80.

Vaillancourt, Lorraine et Cloutier, Nathalie (2009), Un parcours contemporain: NEM 1989-2009, Montréal, Nouvel Ensemble Moderne.

1. Le titre de ce texte est une paraphrase de la conclusion de l'entretien réalisé conjointement par Lorraine Vaillancourt et Jonathan Goldman, "Donner le temps: entretien avec Lorraine Vaillancourt". Voir Goldman, 2013, p. 62.

2. Ce texte est une commande conjointe du Nouvel Ensemble Moderne (NEM) et de la Société québécoise de recherche en musique (SQRM), et fut imprimé à l'origine dans le programme du "Grand concert $30^{\mathrm{e}}$ anniversaire" de l'ensemble, lequel eut lieu le 3 mai 2019 à la salle Claude-Champagne de la Faculté de musique de l'Université de Montréal. Ce concert a d'ailleurs reçu le prix Opus du Conseil québécois de la musique "Concert de l'année - Musiques moderne, contemporaine", remis le 19 janvier 2020. L'auteur et la revue Circuit remercient le NEM et la SQRM d'autoriser la reproduction de ce texte à des fins de mémoire.

3. Kozin, 1990. 